\title{
LEXIKON LITERATURVERFILMUNGEN
}




\title{
LEXIKON LITERATUR- VERFILMUNGEN
}

\author{
Deutschsprachige Filme \\ 1945 - 1990
}

zusammengestellt von

Klaus M. und Ingrid Schmidt

Verlag J.B. Metzler

Stuttgart - Weimar 
Die Deutsche Bibliothek - CIP-Einheitsaufnahme

Schmidt, Klaus M.:

Lexikon Literaturverfilmungen : deutschsprachige Filme 1945 - 1990 /

zsgest. von Klaus M. und Ingrid Schmidt. - Surrgart ;

Weimar: Metzler, 1995

ISBN 978-3-476-01298-2

NE: Schmidt, Ingrid:; HS

ISBN 978-3-476-01298-2

ISBN 978-3-476-03592-9 (eBook)

DOI 10.1007/978-3-476-03592-9

Dieses Werk einschließßlich aller seiner Teile ist urheberrechtlich geschützt. Jede Verwertung außerhalb der engen Grenzen des Urheberrechtsgesetzes ist ohne Zustimmung des Verlages unzulässig und strafbar. Das gilt insbesondere für Vervielfältigungen, Übersetzungen, Mikroverfilmungen und die Einspeicherung und Verarbeitung in elektronischen Systemen.

\section{1995 Springer-Verlag GmbH Deutschland}

Ursprünglich erschienen bei J. B. Metzlersche Verlagsbuchhandlung

und Carl Ernst Poeschel Verlag GmbH in Stuttgart 1995 


\section{Einleitung}

Das vorliegende Werk ist das Ergebnis langjähriger Sammelarbeit aus Archiven, Nachschlagewerken und Zeitschriften. Besonderer Dank sei Herrn Joachim Klünder vom Rundfunk- und Fernseharchiv in Frankfurt für die Benutzung der Originalunterlagen ausgesprochen, sowie Herrn Gunther Scholz, mit dessen Hilfe uns zusätzliche Daten der DEFA zur Verfügung gestellt wurden. Die Sammelarbeit zu diesem Lexikon erfolgte mit Hilfe einer elektronischen Datenbank, wodurch eine rasche Sortierung und Indizierung des Materials möglich wurde. Es ist aufgeteilt in vier Indizes, Autorenindex, Index zu den Buchtiteln, Filmtitelindex und Index zu den Regisseuren und Regisseurinnen. Damit ist der Informationseinstieg von allen Richtungen her gewährleistet, wobei der Autorenindex die vollständigsten Informationen enthält. Aber auch bei den Indizes zu Buch- und Filmtiteln und zum Verzeichnis der Regisseure wurden die wichtigsten Informationen erhalten, damit ein zu häufiges Rückblättern auf den Autorenindex nicht notwendig ist.

Wie jedes Sammelwerk dieser Art ist auch das vorliegende unvollständig. Dies gilt besonders für die Daten über Schriftsteller, die der sogenannten populären oder der Jugend- und Kinderliteratur angehören und deshalb leider nur sporadisch in einschlägigen Literaturlexika und biographischen Nachschlagewerken vorkommen. Hier stießen wir an Grenzen, da weitere Angaben nur sehr schwer zugänglich waren. Trotzdem hielten wir es für außerordentlich wichtig, daß bei einem Nachschlagewerk wie dem unseren keine willkürlichen Grenzen zwischen Werken der sogenannten Hochkultur und der populären Literatur oder der Kinder- und Jugendliteratur gezogen wurden. Im vorliegenden Lexikon sind solche großräumigen Gattungsunterscheidungen, wenn überhaupt, nur an Genrebezeichnungen wie „Kriminalroman“ oder „Kinderbuch“ zu erkennen. Weitere Grenzziehungen sollen, wenn sie partout für sinnvoll gehalten werden, dem Benutzer überlassen sein.

Eine systematische Erfassung der Literaturverfilmungen erfolgte für den Zeitraum 1945 -1990. Dieser bietet sich als geschlossene Einheit zwischen dem Ende des zweiten Weltkrieges und den politischen Umwälzungen, die zur Zeit generell als „Wende" bezeichnet werden, an. Als allgemeines Kriterium für die Aufnahme galt nicht etwa die Beschränkung auf deutschsprachige Filmemacher, Drehbuchschreiber oder Darsteller sondern einzig und allein, ob ein Werk in einem deutschsprachigen Land produziert oder zumindest koproduziert wurde. Der deutschsprachige Raum beschränkt sich auf die Länder Bundesrepublik Deutschland, Deutsche Demokratische Republik, Republik Österreich und Schweiz. So taucht zum Beispiel die von Volker Schlöndorff für das amerikanische Fernsehen produzierte Bearbeitung von Arthur Millers Tod eines Handlungsreisenden in diesem Lexikon nicht auf. Dagegen haben wir uns erlaubt, gelegentlich ein Werk aus der früheren oder späteren Periode mit aufzunehmen, wenn es dem Informationszusammenhang dient. Dasselbe gilt auch für einige wenige Filme, die im nicht deutschsprachigen Raum produziert wurden. Zum Beispiel wird Luchino Viscontis Verfilmung von Thomas Manns Tod in Venedig in unserem Werk aufgeführt, um einen möglichst vollständigen Überblick der Thomas Mann-Verfilmungen zu gewährleisten. Ebenfalls aus Gründen der Vollständigkeit haben wir auch Beispiele von Literarisierungen aufgenommen, das sind literarische Bearbeitungen von Filmen, die oft im Zuge der gemeinsamen Vermarktung von Film und Literatur entstanden sind. Neben ei- 
nigen auf diese Weise entstandenen Kriminalromanen ist eines der bekanntesten Beispiele die Literarisierung des Erfolgsfilms von Fassbinders Die Ehe der Maria Braun durch Gerhard Zwerenz. Zu erkennen sind solche Literarisierungen oft an dem späteren Erscheinungsdatum des Buches gegenüber dem Film. Allerdings waren diese Daten nicht immer zuverlässig eruierbar. Bei Verfilmungen von dramatischer Literatur haben wir uns bemüht, nur solche Produktionen aufzunehmen, die auch speziell als Film konzipiert wurden, also keine Aufzeichnungen von Theaterinszenierungen. Aber auch dabei gibt es Grenzfälle wie etwa die Fernsehaufzeichnung von Fassbinders Theaterinszenierung seiner Bremer Freiheit oder die Verfilmung von Gründgens' Faustinszenierung. Im Zweifelsfalle wurde eine Produktion eher aufgenommen als weggelassen.

Eine gesonderte Behandlung von Kinofilmund Fernsehproduktionen haben wir bewußt vermieden, nicht nur weil durch das komplizierte Kooperationssystem zwischen Kinoproduktion und Fernsehen besonders in der BRD die Grenzen oft fließend sind, sondern weil damit ebenfalls der Gesamtüberblick etwa in bezug auf Autoren, Regisseure, Bearbeitung bestimmter Titel, etc. verlorengegangen wäre. Dennoch ist im Hauptindex zu den Autorinnen und Autoren jede Verfilmung, sofern die Information zugänglich war, als Kino-oder Fernsehfilm gekennzeichnet.

Neben der allgemeinen alphabetischen Ordnung gilt für jeden einzelnen Lexikonartikel die chronologische Reihung. Bei Doppelautoren sind die Lebensdaten, wenn überhaupt für beide vorhanden, in der Reihenfolge ihres Erscheinens im Artikel gegeben und jeweils durch (/) Schrägstrich getrennt. Wir haben uns bemüht, bei ausländischer Literatur auf die Originaltitel in transkribierter Form zu- rückzugehen, was besonders bei kleineren Erzählungen nicht immer möglich war. Stand nur der deutsche Buchtitel zur Verfügung, so wird dieser in Klammern aufgeführt. Bei einer Anzahl von Verfilmungen konnte kein literarischer Titel mehr ermittelt werden. In den meisten Fällen ergab sich aber zumindest ein Hinweis auf das verfilmte literarische Genre. Genrebezeichnungen wurden nach Möglichkeit vereinheitlicht. So wurden Bezeichnungen wie „Lustspiel“, „Schwank“, „Farce“, etc. unter der Rubrik „Komödie“ vereinheitlicht. Auch Verfilmungen von Opern und Operetten wurden in das Lexikon aufgenommen, wenn die Angaben auch nicht immer deutlich nach Verfasser von Libretto und Komponist getrennt sind. Die Titel der Vorlage sind, wenn zugänglich, in der Originalsprache zitiert, und die deutschen Übersetzungen wurden in Klammern gesetzt. Erscheinungsdaten beziehen sich bei Literaturtiteln auf die Erstpublikation, bei Filmen auf die Spielzeiteröffnung im Kino oder auf die Erstsendung. Allerdings waren diese Daten nicht immer zuverlässig zu erbringen. Bei Regisseurinnen und Regisseuren haben wir auf die Angabe von Herkunftsland und Lebensdaten verzichtet, da für Regisseure, die überwiegend fürs Kino gearbeitet haben, diese Information leicht den einschlägigen Filmlexi$\mathrm{ka}$ entnommen werden können. Bei reinen Fernsehregisseuren stehen, wo immer möglich, Informationen zum Herkunftsland und der Sendeanstalt der jeweiligen Fernsehproduktion zur Verfügung.

Mit diesem Lexikon wollen wir ein längst überfälliges Arbeitsinstrument für Literaturund Medienwissenschaftler, Filmhistoriker und allgemeine Film- und Literaturfans zur Verfügung stellen. Wertungen und Deutungen überlassen wir den Benutzerinnen und Benutzern. Einige Statistiken zum vorliegenden Material sollen die Arbeit weiter erleichtern. 


\section{Statistischer Überblick}

\section{Anzahl der Produktionen von Literaturverfilmungen}

\author{
$1945-1949$ \\ $1950-1959$ \\ $1960-1969$ \\ $1970-1979$ \\ $1980-1989$ \\ 1990 - \\ Ohne Datum \\ Kinoproduktionen \\ Fernsehproduktionen \\ Insgesamt erfaßt
}

Spanien

Belgien

Argentinien

43

867

1245

918

720

27

14

1259

2575

3834

Rumänien

Brasilien

Columbien

Griechenland

Uruguay

Chile

Portugal

Insgesamt

\section{Verfilmungen nach Herkunftsland und Sprachraum der literarischen Vorlage}

\begin{tabular}{l}
\multicolumn{2}{c}{ Vorwiegend deutschsprachiger Raum } \\
Bundesrepublik Deutschland \\
Deutschland vor 1945 \\
Österreich \\
Deutsche Demokratische Republik \\
Schweiz \\
Liechtenstein \\
Insgesamt \\
\multicolumn{2}{l}{ Vorwiegend englischsprachiger Raum }
\end{tabular}

Großbritannien

\section{7}

Vereinigte Staaten von Amerika

Irland

Canada

Australien

Rhodesien

Insgesamt

Frankreich

Romanischer Sprachraum

Italien

777

628

382

355

130

2

2274

\section{2}

26

9

6

5

2

2

2

2

\section{Slavischer Sprachraum}

Russland (vor der Revolution)

Tschechoslovakei

Sowjetunion 47

Polen 31

Bulgarien 2

Jugoslawien 2

Litauen 1

Insgesamt $\mathbf{2 1 6}$

\section{Skandinavischer und niederländischer Sprachraum}

Schweden $\quad 30$

Dänemark 26

Norwegen 21

Niederlande $\quad 12$

Island 2

Insgesamt $\quad 91$

Sonstige Sprachräume

709 Türkei 4

Finnland 1

Indien 1

332 Insgesamt $\quad 67$

51 Ohne Angabe 40 


\section{Verfilmungen nach literarischen Genres ${ }^{1}$}

Epische Formen
Roman
Erzählung
Autobiografie
Bericht
Bildergeschichte
Reportage
Briefe
Biografie
Essay
Tagebuch
Epos
Texte
Chronik
Dokumente
Sachbuch
Volksbuch
Dramatische Formen
Schauspiel
Komödie
Hörspiel
Musikkomödie
Operette
Volksstück
Dialog
Monolog
Oper
Märchenspiel

$\begin{array}{rlr}\mathbf{1 8 0 1} & \text { Musical } & 2 \\ 1096 & \text { Musikspiel } & 2 \\ 628 & \text { Schattenspiel } & 1 \\ 23 & \text { Szenen } & 1 \\ 16 & \text { Szenario } & 1 \\ 6 & & \\ 5 & \text { Lyrische Formen } & \mathbf{9} \\ 5 & \text { Gedicht } & 6 \\ 4 & \text { Ballade } & 3 \\ 4 & & \\ 4 & \text { Kinder- und Jugendliteratur } & \mathbf{2 2 0} \\ 4 & \text { Märchen } & 97 \\ 2 & \text { Kinderbuch } & 37 \\ 1 & \text { Kindererzählung } & 28 \\ 1 & \text { Kinderroman } & 7 \\ 1 & \text { Jugendbuch } & 37 \\ 1 & \text { Jugenderzählung } & 7 \\ & \text { Jugendroman } & 3 \\ \mathbf{1 6 1 0} & \text { Märchenspiel } & 3 \\ 939 & \text { Jugendstück } & 1 \\ 540 & & \\ 67 & \text { Kriminalliteratur } & \mathbf{1 7 5} \\ 20 & \text { Kriminalroman } & 125 \\ 15 & \text { Kriminalstück } & 27 \\ 7 & \text { Kriminalkomödie } & 13 \\ 4 & \text { Kriminalerzählung } & 10 \\ 4 & & \mathbf{4 4} \\ 4 & & \\ 3 & \text { Ohne Angabe } & \end{array}$

1 Bei den Genreangaben ist zu berücksichtigen, daß einige Doppelzählungen auftreten, und zwar wenn etwa eine Verfilmung auf einem Schauspiel beruht, das wiederum die dramatische Bearbeitung eines Romanes ist. 


\section{Abkürzungen von Ländernamen:}

\begin{tabular}{|c|c|c|c|c|c|}
\hline ARG & $=$ & Argentinien & JUG & $=$ & Jugoslawien \\
\hline AUST & $=$ & Österreich & LIE & $=$ & Liechtenstein \\
\hline AUSTRAL & $=$ & Australien & LIT & $=$ & Litauen \\
\hline BELG & $=$ & Belgien & NL & $=$ & Niederlande \\
\hline BRAS & $=$ & Brasilien & NOR & $=$ & Norwegen \\
\hline BRD & $=$ & Bundesrepublik Deutschland & PL & $=$ & Polen \\
\hline BULG & $=$ & Bulgarien & PORT & $=$ & Portugal \\
\hline CAN & $=$ & Canada & RHOD & $=$ & Rhodesien \\
\hline $\mathrm{CH}$ & $=$ & Schweiz & RU & $=$ & Rumänien \\
\hline CHIL & $=$ & Chile & RUS & $=$ & Rußland (vor 1917) \\
\hline $\mathrm{COL}$ & $=$ & Columbien & SU & $=$ & Sowjetunion \\
\hline CSR & $=$ & Tschechoslowakei & SVE & $=$ & Schweden \\
\hline DDR & $=$ & Deutsche Demokratische & TUR & $=$ & Türkei \\
\hline & & Republik & URUG & $=$ & Uruguay \\
\hline DK & $=$ & Dänemark & USA & $=$ & Vereinigte Staaten \\
\hline DTL & $=$ & Deutschland (vor 1945) & & & von Amerika \\
\hline ESP & $=$ & Spanien & ZIMB & $=$ & Zimbabwe (Rhodesien) \\
\hline FR & $=$ & Frankreich & & & \\
\hline FIN & $=$ & Finnland & \multicolumn{3}{|c|}{ Sonstige Abkürzungen: } \\
\hline GB & $=$ & Großbritannien & & & \\
\hline HEL & $=$ & Griechenland & $\mathrm{A}$ & $=$ & Autor \\
\hline HU & $=$ & Ungarn & BT & $=$ & Buchtitel \\
\hline IND & $=$ & Indien & DB & $=$ & Drehbuch \\
\hline IRL & $=$ & Irland & FT & $=$ & Filmtitel \\
\hline ISL & $=$ & Island & $\mathrm{KF}$ & $=$ & Kinofilm \\
\hline ISR & $=$ & Israel & $\mathrm{R}$ & $=$ & Regisseur \\
\hline IT & $=$ & Italien & TV & $=$ & Fernsehfilm \\
\hline
\end{tabular}




\section{Erklärungen zum Autorenregister:}

Böll, Heinrich (BRD 1917-1985) = Autor mit Herkunftsland und Lebensdaten

Bouchaud, Jean = Weder Herkunftsland noch Daten des Autors bekannt

Sjöwall, Mal \& Wahlöö, Per (SVE 1935-/ 1926-75) =

Lebensdaten in der Reihenfolge der Autoren

\section{Verhoeven, Paul \& Nick, Edmund \&}

Impekhoven, Toni (DTL--/1891-?/--) = Nur Geburtsdatum des mittleren Autors bekannt

Kräly, Hans (BRD?-1950) = Nur Todesjahr bekannt

Beckmann, Heinz (DDR) = Daten des Autors nicht bekannt

Charpentier, Jules (= Josef \& Berta Engelsberger) $=$

Name ist Pseudonym, richtiger Name in Klammern

Harvey $(1945)($ Mein Freund Harvey 1950) $=$ Originaltitel und Titel der deutschen Übersetzung mit Erscheinungsjahr

Mission to Venice $(1954)(\mathrm{dt} .1964)=$ Originaltitel mit Erscheinungsjahr, Titel der deutschen Übersetzung nicht bekannt, aber Erscheinungsjahr

Hét pofon (Sieben Ohrfeigen 1962) = Nur das Erscheinungsjahr der Übersetzung bekannt

(Die Grenzziehung) $($ Ges. Werke 1981) $=$ Nur deutscher Titel bekannt, erschienen innerhalb der gesammelten Werke

(Das Jubiläum $)($ Komödie $)=$

Originaltitel nicht bekannt; deutscher Titel nicht bestätigt (Quelle sagt: ,nach der gleichnamigen Komödie“)

Wir sind Utopia $(1943)(>$ Gottes Utopia 1950) $=$ Buch erschien später unter neuem Titel

Die Feuerzangenbowle (1935) (Roman)

$(>$ Komödie 1938) $=$
Ursprünglich als Roman, später als Komödie erschienen

Der Prozeß der Jeanne d'Arc zu Rouen 1431 $(<$ nach Seghers $)=$

Buch basiert auf einem Buch von anderem $/ \mathrm{r}$ Autor/in

Look homeward, Angel (1958) (Schau heimwärts, Engel) (Schauspiel < Wolfe, Thomas:

Look Homeward, Angel (1929); (Schau heimwärts, Engel (1932) (Roman)) =

Schauspiel (ersch. 1958) basierend auf einem Roman von Thomas Wolfe (ersch. 1929, dt. Übers. 1932)

Hoffman (Shall I Eat You Now?, brit.) (1969) = Britische Ausgabe unter anderem Titel

A la recherche du temps perdu (1913) (Kapitel) $($ Roman $)=$

Ein Kapitel aus dem Roman verfilmt

Die Räuber (1781) (Schauspiel) (frei nach) $=$ Freie Bearbeitung des Werkes

(Roman) R: Vohrer, Alfred =

Genre bekannt, aber kein Buchtitel

Hochzeitsreise (Schauspiel) R: Glück, Wolfgang =

Buchtitel und Genre bekannt, aber kein

Erscheinungsjahr

Herr Korbes empfängt (1957) (Komödie<Hörspiel) $=$

Erschien zuerst als Hörspiel

Ende der Anfrage (Essay/Hörspiel <Dokumentarbericht) $(1967)=$

Erschien zuerst als Dokumentarbericht, dann als Essay und Hörspiel

R: Sydow, Rolf v.; FT: Wohl bekomm's (1982) = Weder Buchtitel noch Genre bekannt

FT: Das Boot (1981); KF/TV: BRD/ARD =

Sowohl als Kinofilm als auch als Fernsehfilm erschienen

FT: Bolwieser (2 Teile 1976/77)(1983); TV/

$\mathrm{KF}$ : BRD/Bavaria/ZDF =

Erschien zuerst als 2-teiliger Fernsehfilm, dann als Kinofilm 